
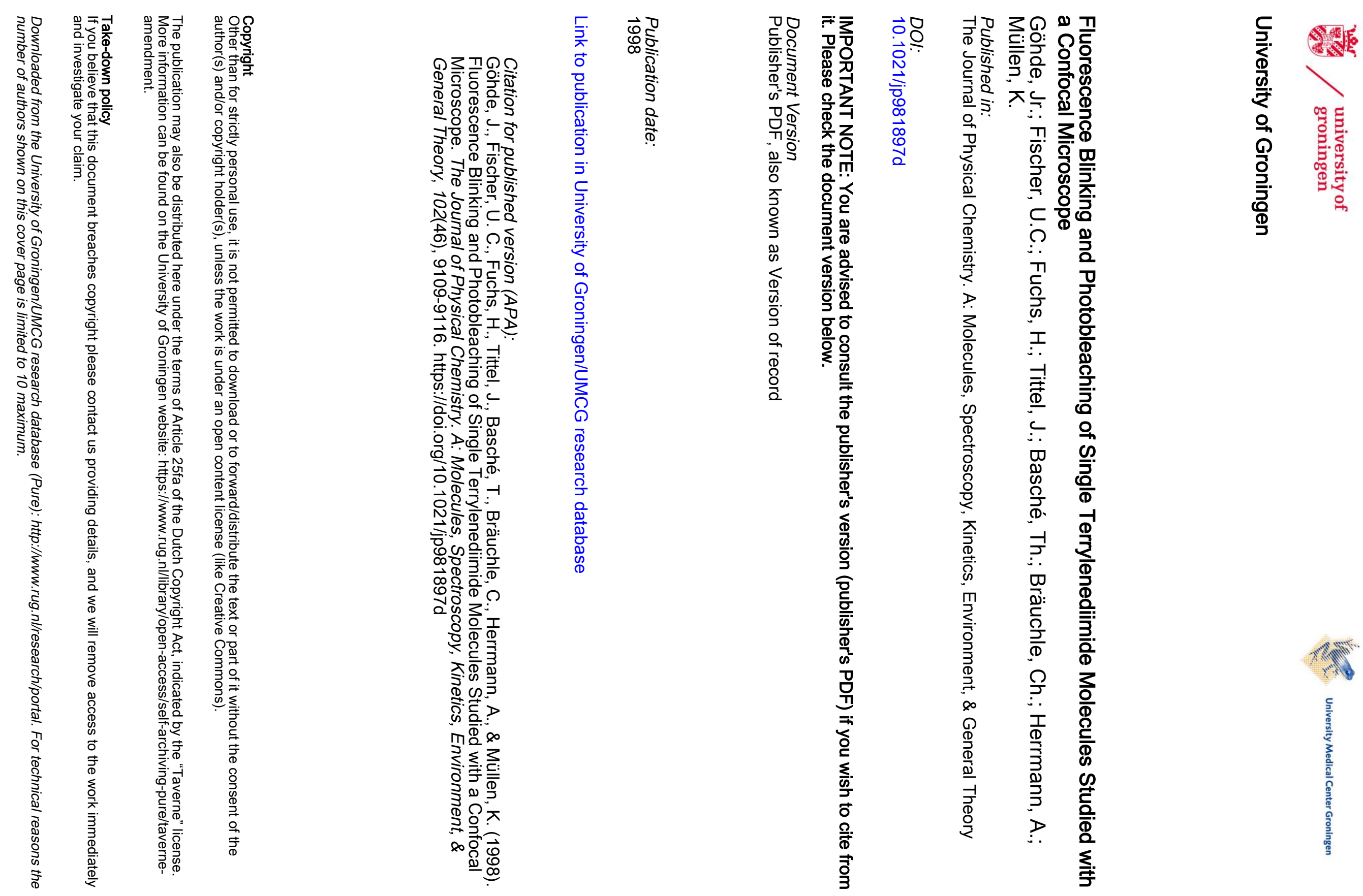


\title{
Fluorescence Blinking and Photobleaching of Single Terrylenediimide Molecules Studied with a Confocal Microscope
}

\author{
W. Göhde, Jr., U. C. Fischer, and H. Fuchs \\ Physikalisches Institut, Westfälische Wilhelms-Universität Münster, D-48149 Münster, Germany \\ J. Tittel, Th. Basché, and Ch. Bräuchle \\ Institut für Physikalische Chemie, Ludwig-Maximilians-Universität München, D-80333 München, Germany
}

\author{
A. Herrmann and K. Miillen \\ Max-Planck-Institut für Polymerforschung, D-55128 Mainz, Germany
}

Received: April 16, 1998; In Final Form: June 9, 1998

\begin{abstract}
Single terrylenediimide molecules diluted in a 20-nm-thick polyvinylbutyral polymer film were localized and observed by scanning confocal fluorescence microscopy. A modular and compact confocal microscope and the high optical stability of the molecules allowed a repeated imaging and observation over $>5 \mathrm{~h}$ at room temperature. Most of the molecules showed several "on-off-on" transitions (blinking) on a time scale from seconds to hours, before permanent bleaching occurred. We determined that $>1.5 \times 10^{7}$ fluorescence photons are emitted from the most-stable molecules before the final bleaching step occurs. Despite the "onoff-on" transitions, however, the overall change in fluorescence intensity, either integrated over each image of a time series or summed for several individual molecules, resembled an exponential-like decay, familiar from measurements of many-molecule ensembles. We also observed the polarization of the fluorescence from single molecules during excitation with circular polarized light. From these measurements, possible rotations of the molecular dipoles were studied. Over a span of $5 \mathrm{~h}$, the polarization angle in most cases did not change by $>15-20^{\circ}$. This may explain the slow and small intensity changes but excludes molecular rotation as a reason for the blinking behavior.
\end{abstract}

\section{Introduction}

The microscopic observation of fluorescence from single chromophores adsorbed on surfaces or in thin films or membranes $^{1-7}$ and in fluids ${ }^{8,9}$ at room temperature and low temperatures ${ }^{10,11}$ gives direct insight into several processes that are not accessible in ensemble measurements, where fluorescence is detected from many molecules simultaneously. For example, bleaching of a single molecule can appear in one step ${ }^{2,4}$ after several fluorescence cycles, the number of which may vary statistically between different molecules, whereas a fluorescence bleaching curve of a molecule ensemble decays continuously and nearly exponentially in time. Variations from a singleexponential bleaching curve may be related to the random orientation within the ensemble or to inhomogeneities in the individual local environment of each molecule. The investigation of single-molecule fluorescence is expected to uncover such inhomogeneities on a molecular scale. Apart from permanent bleaching, new effects have already been observed on the singlemolecule level, such as large, photoinduced spectral shifts ${ }^{12,13}$ and, possibly related to this, a sudden reappearance of fluorescence some time after an instantaneous drop of the fluorescence intensity. ${ }^{2,4,14}$ One fascinating application of microscopic single- molecule fluorescence detection is the observation and localization of chemical reactions on the single-molecule level. ${ }^{15,16}$

To obtain insights that are statistically relevant, one must collect data from many molecules. Conventional scanning and nonscanning microscopy, combined with detectors with high photon counting efficiencies such as cooled avalanche photodiodes $^{1}$ and cooled charge-coupled device (CCD) cameras, ${ }^{7,11}$ are simple and reliable techniques for these investigations. Scanning near-field optical microscopy (SNOM), first used for imaging single dye molecules on surfaces, ${ }^{1}$ offers resolution superior to that of conventional far-field microscopy. Furthermore, because of the smaller excitation volume, SNOM should, in principle, decrease the background level against which the single molecule fluoresceces. However, at present production of coated optical-fiber-tip probes with apertures suited for singlemolecule detection is difficult, making SNOM more cumbersome to use in this case than conventional microscopes. With a combined confocal and near-field optical microscope, ${ }^{17}$ we compared the confocal mode with the near-field mode. The resolution in the confocal mode is only about a factor of five worse than that of the SNOM mode of 50-150 nm but offers other important advantages: (1) better suppression (by 10-fold) of the excitation light because of the epi-illumination scheme; 


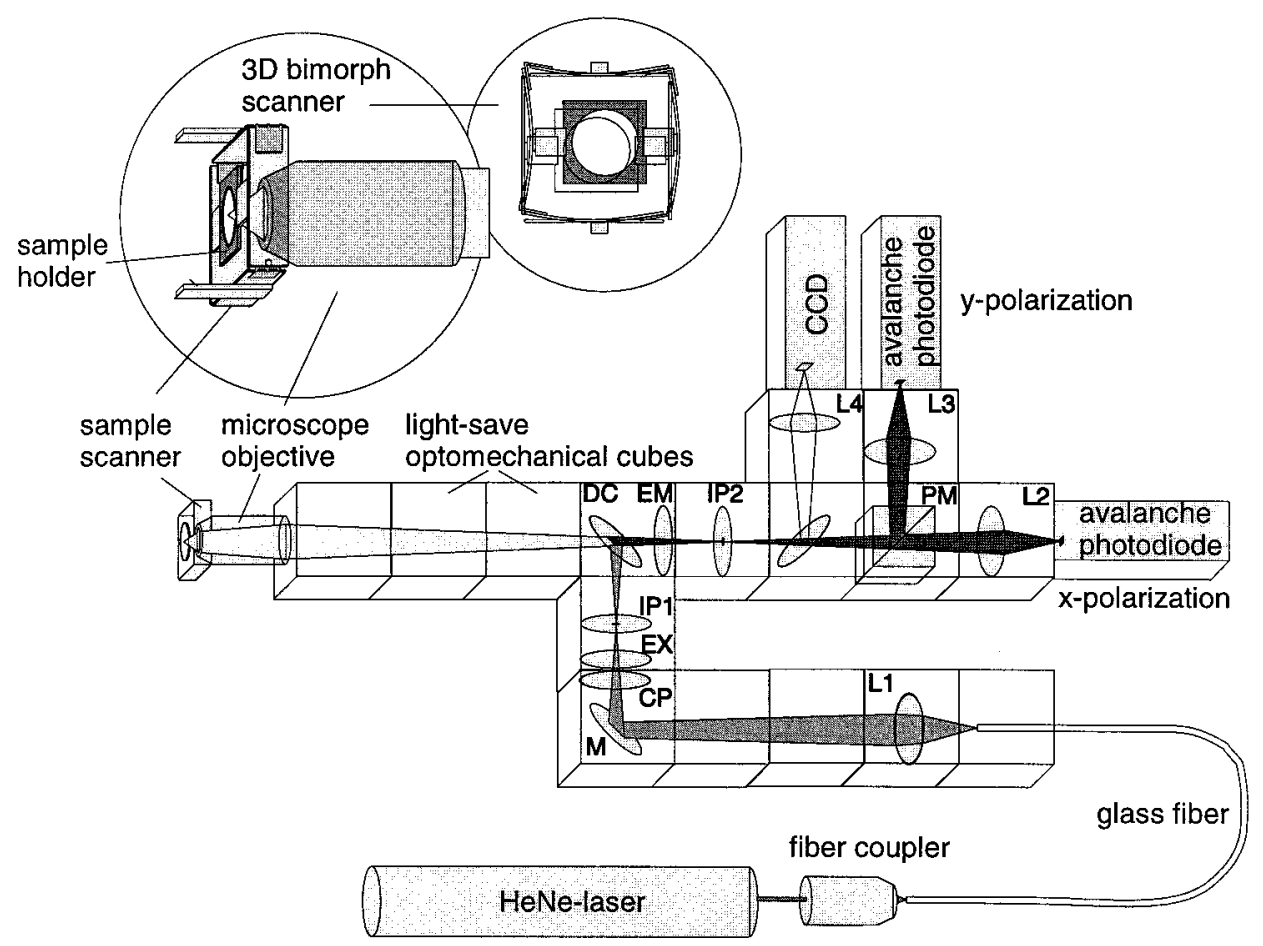

Figure 1. Optical setup of the modular confocal microscope. Insets: Details and arrangement of wide-range three-dimensional (3D) bimorph sample scanner and objective. L1-L4, lenses; M, mirror; CP, circular polarizator; EX, excitation filter; IP1, confocal pinhole (excitation); DC, dichroic mirror; EM, emission filter; IP2, confocal pinhole (emission); PM polarizing beamsplit. The CCD camera is used for adjustments.

(2) the possibility of higher excitation intensities than with the fiber tips; (3) three-dimensional imaging capability; and (4) ease of use. Therefore, for investigations of samples with diluted molecules, we found the confocal mode to be much better suited than SNOM.

Here we report on fluorescence bleaching experiments with terrylenediimide (TDI) molecules ${ }^{18}$ embedded in a polyvinylbutyral (PVB) polymer film. These dye molecules, excited by a continuous HeNe laser line at $633 \mathrm{~nm}$, allow quantitative analysis of single-molecule fluorescence because of their high photostability and high fluorescence quantum yield. The fluorescence intensity of single molecules measured in the confocal microscope depends on the molecular orientation and, for individual molecules, differs between zero and some maximum value. We compared the influence of the orientation of the molecules' dipole moments onto the local intensities in the images with the images obtained by computer simulations. Measurements of the fluorescence polarization were performed to investigate whether molecular rotation took place, which could be a reason for slow intensity changes or blinking.

\section{Experimental Section}

The sample is scanned line by line in the focus of an on-axis confocal microscope by using an $80-\mu \mathrm{m}$-range three-dimensional sample scanner to acquire the fluorescence intensity profiles (Figure 1). A similar instrument has been used for measurements in a low-temperature cryostat ${ }^{19}$ and is described in more detail elsewhere. ${ }^{17}$ These microscopes can also be used in a near-field optical mode to excite single molecules near an aperture of a coated fiber tip, if a subwavelength resolution is required. All measurements discussed here were done in the confocal mode. Our modular microscope is based on optomechanical cubes (partec) mounted onto an optical bench (Figure 1). The cubes are well suited for the setup of a modular microscope because they ensure that no light from the outside enters the optical path, as is obviously crucial for single- molecule experiments, where minute light powers have to be detected. Lenses, filters, diaphragms, and detectors can easily be mounted and switched in and out of position.

Light from a $20 \mathrm{~mW}$ HeNe laser with $\lambda=633 \mathrm{~nm}$ is coupled into the entry of a single-mode fiber. The 4- $\mu \mathrm{m}$-diameter core on the end of the fiber is magnified into one of the intermediateimage planes of the microscope objective, after being attenuated by neutral filters and band-pass-filtered by an excitation filter (633NB3.0, part of Omega "dye set XF46"). By inserting a linear or circular polarizer, one can select linear or nearly circular polarized excitation light. After passing the excitation pinhole, the laser light is reflected into the objective by a dichroic mirror (Omega 645DRLP02) with a corner wavelength of $645 \mathrm{~nm}$. An objective with a numerical aperture of 0.8 (partec $50 \times 0.8$ ) focuses the laser light into the focal plane on-axis to give an excitation spot with a diameter of $0.8-1.2 \mu \mathrm{m}$ at one-half the intensity maximum, as can be deduced from the spots originating from single molecules in the images (to be shown later). The fluorescence of the sample is collected by the same objective and passes the dichroic mirror and an interference band-pass filter (Omega 670DF40), which blocks the excitation light reflected from the sample and objective lenses. A pinhole of $200 \mu \mathrm{m}$ diameter in the intermediate-image plane of the emission light path suppresses background light originating from locations more than $\sim 5 \mu \mathrm{m}$ from the excitation spot. By using of smaller pinholes, the resolution as well as the background suppression could be increased further. However, because of different wavelengths of the fluorescence emitted and the use of objectives that show chromatic aberrations, the loss of fluorescence signal becomes too high when smaller pinholes are used. The detection pinhole is demagnified onto the $0.1 \times 0.1 \mathrm{~mm}^{2}$ sensitive area of an avalanche photodiode (EG\&G SPMC200), which detects fluorescence photons with a counting efficiency of $40 \%$, while the dark count rate is $<4 \mathrm{~s}^{-1}$. The number of photons counted in $5 \mathrm{~ms}$ is registered for each image pixel and displayed in a grayscale image. The total detection efficiency 


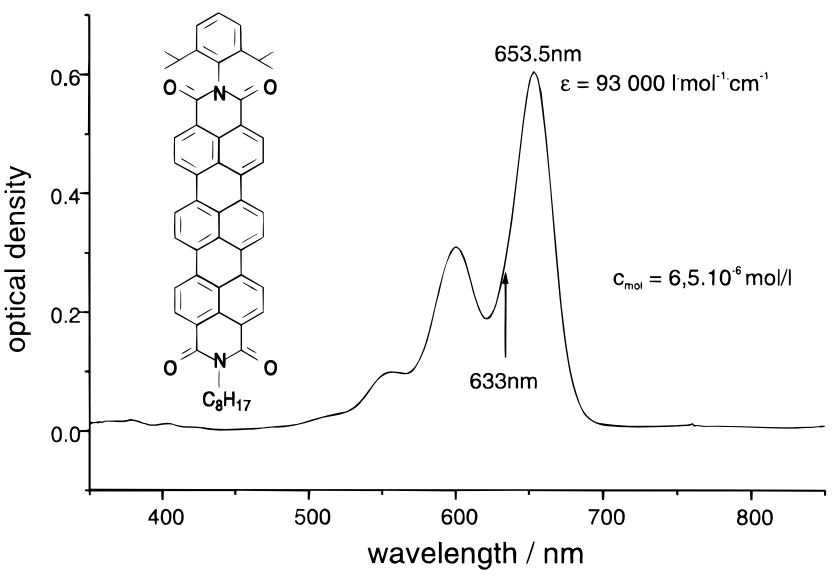

Figure 2. Structure of the TDI molecules ${ }^{19}$ and absorption spectrum in a $\mathrm{CHCl}_{3}$ solution before further dilution. The peak absorptivity at $653.5 \mathrm{~nm}$ is $93000 \mathrm{~L} \cdot \mathrm{mol}^{-1} \cdot \mathrm{cm}^{-1} .18$

of the microscope is estimated to be $1-2 \% .{ }^{17}$ To determine the fluorescence polarization, we used a scheme with two avalanche photodiode detectors (Figure 1), as was also used in by Ruiter et al. ${ }^{5}$ A polarization beam-splitting cube splits the two linear polarization components of the fluorescence onto the photodiodes. The photon signals of both detectors are counted simultaneously by two independent counters. In contrast to the previous study, ${ }^{5}$ we used circular polarized excitation light to excite all in-plane molecular absorption dipoles, rotating or not, with the same strength.

\section{Sample Preparation}

A drop of a solution of TDI ${ }^{18}$ molecules (Figure 2), $0.8 \times$ $10^{-9} \mathrm{~mol} / \mathrm{L}$, and $\mathrm{PVB}, 2 \mathrm{mg} / \mathrm{mL}$, in $\mathrm{CHCl}_{3}$ is spin-coated onto a cleaned quartz cover glass. ${ }^{12}$ This yields homogeneous polymer films $20-30 \mathrm{~nm}$ thick and a calculated average of 12 \pm 6 molecules $/ \mu \mathrm{m}^{2}$. In the fluorescence images, however, only a fraction less than $10 \%$ of this number appears as a spot, as will become clear from the images. By taking into account the photobleaching, working with this concentration makes it convenient to compare and analyze a statistically relevant number of molecules simultaneously in one experiment. The thickness of the films was measured by atomic force microscopy and scanning shear force microscopy by evaluating the depth of the holes that are occasionally present in the films. The dye molecules are well suited for excitation with an inexpensive $\mathrm{HeNe}$ laser or a semiconductor laser diode, given their high absorptivity between 590 and $680 \mathrm{~nm}$ (Figure 2).

\section{Results}

Number of Fluorescing Molecules: Simulation vs Experiment. One of the aims of our numerical simulation was to get a qualitative impression of the fluorescence images that can be obtained from distributions of single molecules. The orientation of the molecular dipole moments plays an important double role concerning the fluorescence signal reaching the detector: first, the excitation rate and, second, the fluorescence detection efficiency strongly depend on the molecular orientation relative to the optical axis. $^{20}$ Under our experimental conditions, excitation and (spontaneous) fluorescence emission are incoherent processes and can be described independently of each other. For the simulation, we used a semiclassical treatment, i.e., describing the electromagnetic field in terms of polarized electromagnetic waves. The excitation light was approximated by a linear polarized plane wave with an Airy-shaped intensity
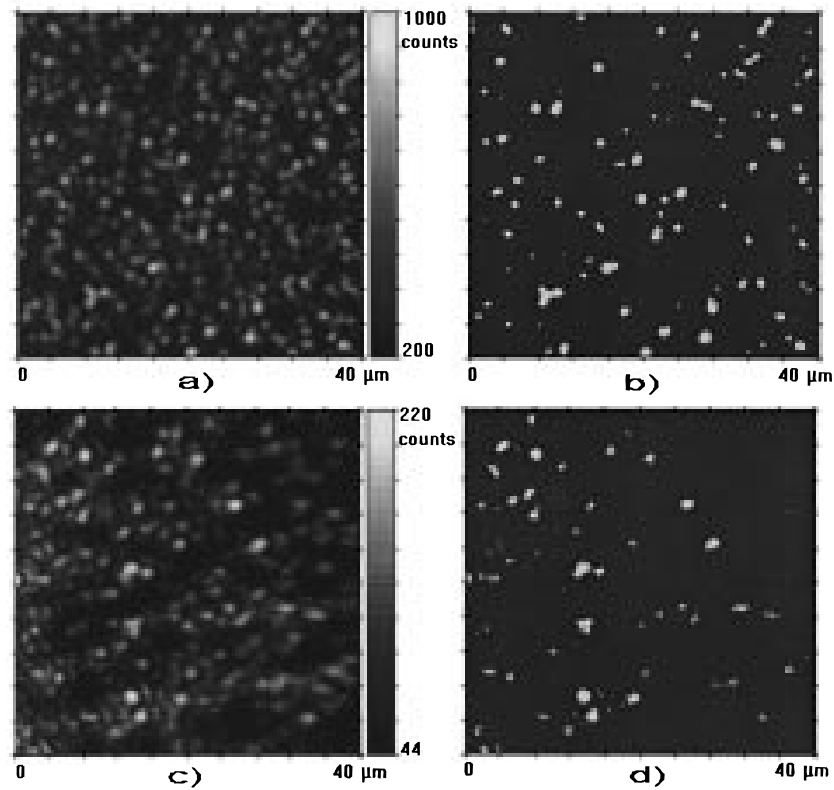

Figure 3. Simulation vs experimental results of confocal single molecule images. (a) Simulation: image area $=40 \mu \mathrm{m} \times 40 \mu \mathrm{m}$. Number of molecules on imaged area, 2000; maximum fluorescence count rate, 1000/pixel; background intensity, 20\%; optical resolution (width of Airy-distribution), $1 \mu \mathrm{m}$. (b) Binary image of simulated image (a). Molecules with an intensity of more than half of the brightest intensity above background in the image are displayed as white spots. Their number, $98 \pm 6$, is only $5 \%$ of the 2000 on the image area. (c) Confocal fluorescence image of TDI in a PVB film at room temperature. (d) Binary image of the experimental data (c) at half of the maximum image intensity above the background. The number of molecular spots in (d) is $57 \pm 10$ or $0.3 \pm 0.2 \%$ of all of the $10000-30000$ molecules on the image area. The concentration of TDI in the PVB film is $6 \pm$ 3 molecules $/ \mu \mathrm{m}^{2}$.

distribution. The width of this distribution corresponds to the size of the laser spot, the lower limit being set by diffraction at the aperture of the objective. Fluorescence excitation and emission as well as photon detection were described as independent stochastic processes; i.e., the quantum statistical probabilities for excitation and emission were used.

Results of the simulation of 2000 molecules on an area of $40 \mu \mathrm{m} \times 40 \mu \mathrm{m}$ made with typical parameters as used in our experiment are shown in Figure 3a. Each spot represents the light from one molecule. The shape of each spot resembles the spatial intensity distribution of the excitation light. Strictly speaking, the molecules, which are small in comparison with the spot width, image the excitation spot. To count molecules from the simulation (Figure 3a), we created a binary black and white image by setting a level such that only spots with an intensity greater than one-half of the maximum count rate appear. As seen in the binary image in Figure $3 \mathrm{~b}$, only $\sim 5 \%$ of the molecules emitted enough fluorescence to appear in the binary image as white spots. We will compare these results with the experimental data later.

For an excitation intensity of $200-400 \mu \mathrm{W} / \mu \mathrm{m}^{2}$ on the optical axis and an acquisition rate of 200 pixels/s, Figure $3 \mathrm{c}$ shows a typical image obtained at room temperature. The scan direction is from bottom to top for each image. All spots in the Figure $3 \mathrm{c}$ have a width of $0.8-1.2 \mu \mathrm{m}$ and are an image of the intensity distribution of the laser spot. As already mentioned, different intensities of the spots can result from different molecular orientations with respect to the excitation polarization and from differences in excitation or emission yields. The counting rate in the brightest spots reaches $300 / 5 \mathrm{~ms}$, which, taking the detection efficiency of $1-2 \%$ into account, corresponds to (3- 

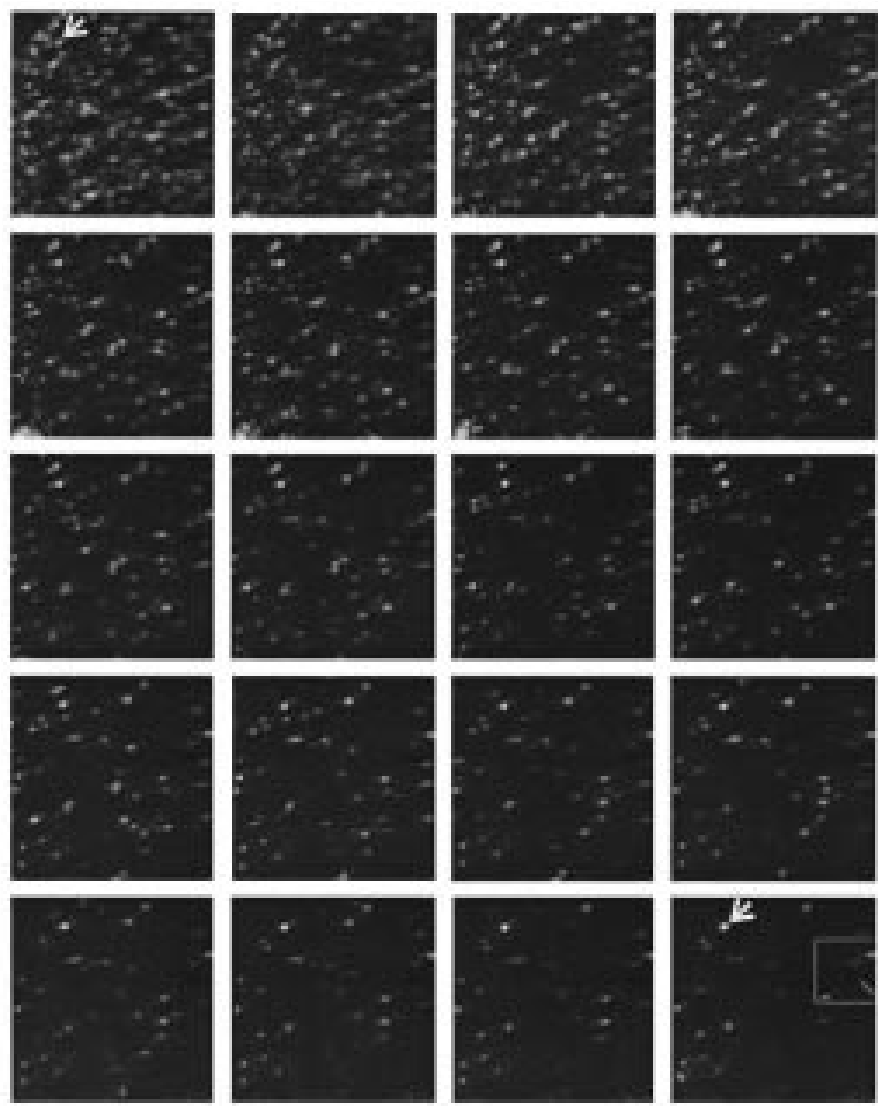
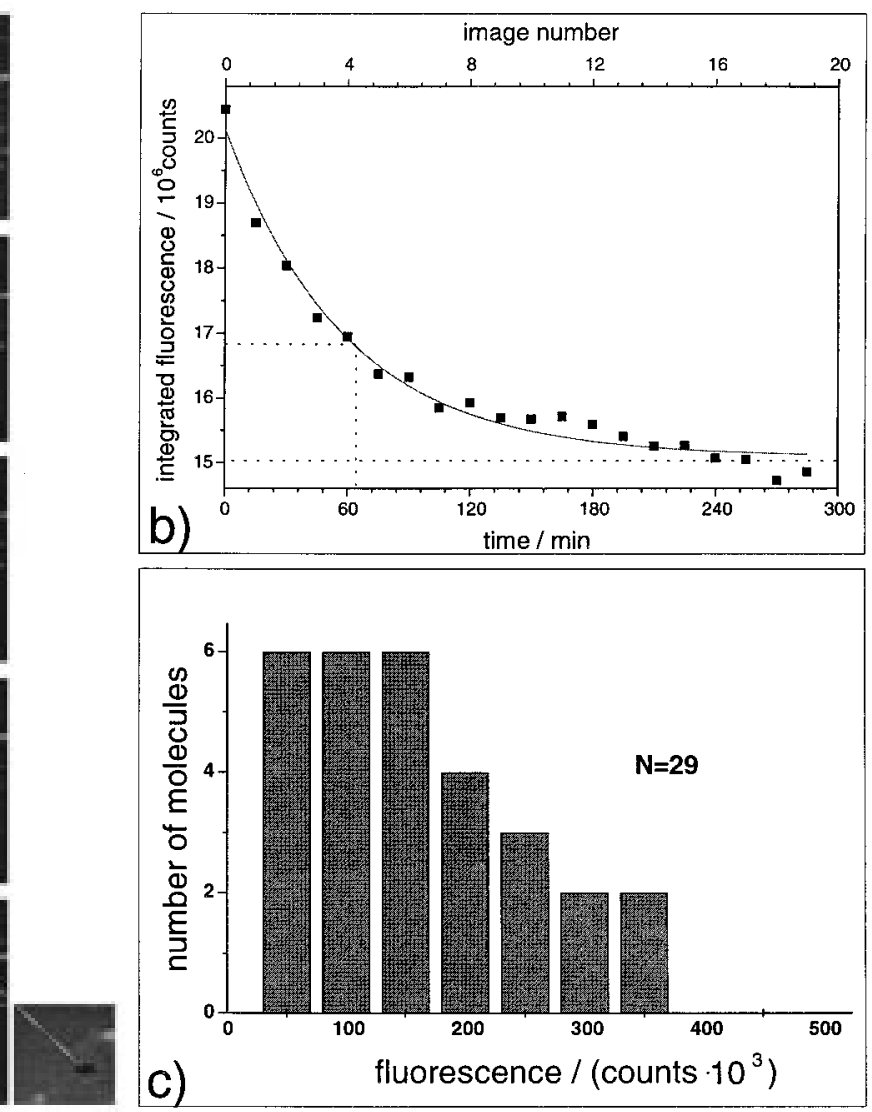

a)

Figure 4. Single-molecule blinking and bleaching and ensemble bleaching in a series of 20 TDI fluorescence images of the same area, taken over a span of $5 \mathrm{~h}$ (a). Some bright molecules (arrows) are stably fluorescing. The time for each image in (a) was 14 min. A black hole in images (zoom at lower right) is due to a hole in the polymer film and shows that the homogeneous background fluorescence of $\sim 150 \mathrm{counts} / 5 \mathrm{~ms}$ originates from the polymer film. (b) Total intensity of each of the images above, showing the bleaching process of the ensemble during repeated imaging. The 1/e time is 64 min. (c) Histogram of the total number of counts obtained for 29 individual molecules.

6) $\times 10^{6}$ fluorescence photons/s. This is 10 -fold less than the saturation rate of TDI of $\mathrm{R}_{\infty}=k_{21}\left(1+k_{\text {isc }} / k_{21}\right) /\left(2+k_{\text {isc }} / k_{\mathrm{T}}\right)=$ $82 \times 10^{6} \mathrm{~s}$, calculated from the transition probabilities given earlier. ${ }^{18,19}$ The corresponding binary image of these data (Figure $3 \mathrm{~d}$ ) shows only $57 \pm 10$ bright spots that exceed onehalf of the maximum intensity in the image (above the background). However, the concentration of dye molecules in the PVB film is $12 \pm 6$ molecules $/ \mu \mathrm{m}^{2}$, and the total number of molecules on the imaged area is $10000-30000$. This ratio of bright spots to the total number of molecules, $0.3 \% \pm 0.2 \%$, is to be compared with the ratio of $5 \%$ between bright molecules and all molecules in the simulated images in Figure $3 b$, where a completely random distribution of the dipole moments was assumed.

With the low-temperature setup, similar images were also acquired at $T=202$ and $96 \mathrm{~K}$ (results not shown). Down to temperatures as low as $96 \mathrm{~K}$, we observed no temperature dependencies, e.g., an increased fluorescence yield. Differences in the counting rates are attributed to slightly different illumination adjustments. From the comparison of the experimental with the simulated data, and the fact that no inhomogeneity of the samples was found on a scale up to a few hundred micrometers, one can be sure that each spot represents no more than a few molecules. In fact, there is a strong evidence that each spot represents the light from exactly one molecule, as will be shown now.

Blinking and Bleaching in Fluorescence Images. Figure 4a shows a series of 20 scans of the same area, taken over $5 \mathrm{~h}$.
In these images, most of the bright spots instantaneously disappear during the first scans, which is characteristic for single molecules bleaching in one $\operatorname{step}^{2,4}$ through transition to a nonfluorescing state, in contrast to the continuous exponentiallike bleaching curve of the fluorescence of an ensemble of many molecules. Two other effects can be seen in the images, or even better, when the images are combined in sequence to make a movie: First, some spots reappear after having disappeared for some time, or show dark stripes, demonstrating a discrete "on-off-on" switching process. This blinking is a characteristic effect in the fluorescence light of single molecules. Second, the intensity of some spots, in comparison with the others, changes slowly between the images. During the 5-h experiment, we observed no diffusion out of a range of $200 \mathrm{~nm}$. Plotting the intensity of each of the 20 images, integrated over the whole image against the image number or time, yielded a nearly exponentially decaying curve for bleaching, as would be expected for a molecule ensemble (Figure 4b). A slight variation of the experimental data from an exact exponential decay is to be expected because of the limited number of molecules contributing to the total intensity and the possible inhomogeneities in the various surrounding molecules. Another effect not considered here are differences in the excitation rates for the randomly oriented molecules, for which the bleaching decay of the ensemble would be expected to be a sum of decaying exponentials rather than a single exponential.

The high background intensity in the bleaching curve, compared with the fairly good signal-to-background ratio (in 

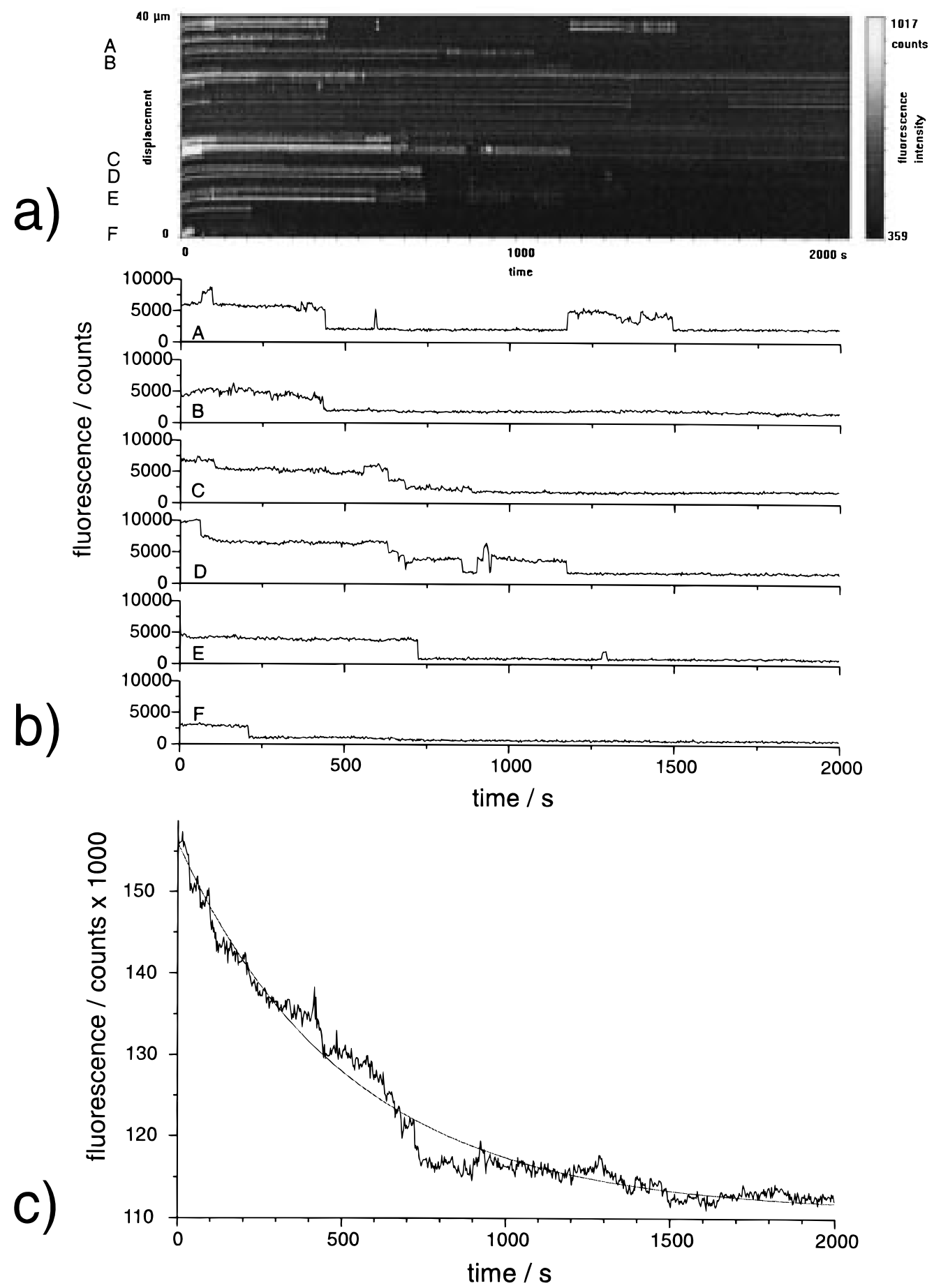

Figure 5. (a) Time-line scans of TDI molecules. Continuous lines represent molecules fluorescing continuously. Broken traces are due to "onoff" transitions of the fluorescence. (b) Traces at positions A-F (integrated over 8 pixels), showing discrete intensity jumps and appearent "inhomogeneities" between individual molecules in the time-dependence of their fluorescence. Some traces show a second or third level of intensity, which could be due to two or three molecules simultaneously emitting fluorescence during some period. The traces are translated against each other for comparison. (c) Fluorescence of (a) integrated over each scan line (256 pixels); the resulting trace resembles a nearly exponential bleaching curve (smooth curve), as known for a molecule ensemble.

Figure 4a) of 5:1 (related to the counts in single pixels), is a consequence of the small overall number of fluorescing dye molecules in the imaged volume of $20 \mathrm{~nm} \times 40 \mu \mathrm{m} \times 40 \mu \mathrm{m}$, compared with the number of PVB molecules, which contribute to the background. The ratio of TDI molecules to PVB molecules is on the order of $10^{3}: 10^{10}$. In experiments with shear force and atomic force microscopes, holes were sometimes detected in the PVB film. The black spot in the background in Figure $4 \mathrm{a}$ is interpreted to indicate such a hole in the polymer film. This is a hint that impurities in the PVB film or in the
PVB itself contribute to the fluorescence signal more strongly than does the nonideal excitation light suppression by the filters. In the series of the 20 images, some molecules remain fluorescing during the experiment. Interestingly, some of these "most-stable" molecules (arrows) are comparatively bright, emitting, in total, many more fluorescence photons than other dim but fast-bleaching molecules.

Photostability of TDI Molecules. Figure $4 \mathrm{c}$ is an evaluation of $N=29$ molecules from which the total number of counts obtained in the 20 images in Figure 5a are classified into a 


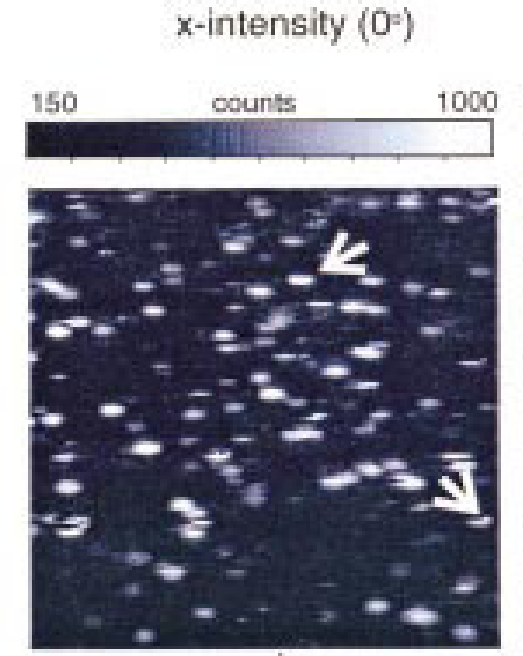

a) y-intensity $\left(90^{\circ}\right)$

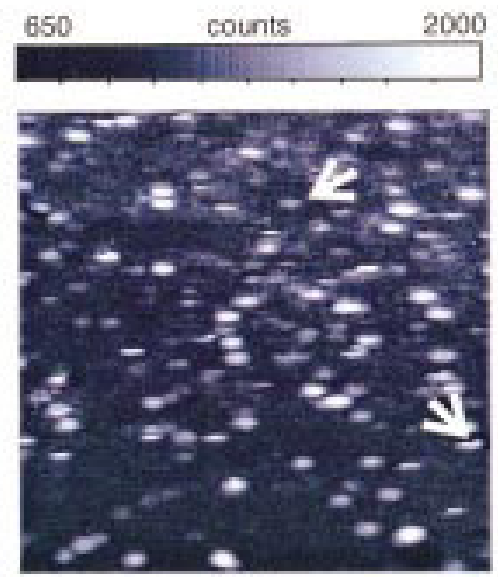

b) polarization
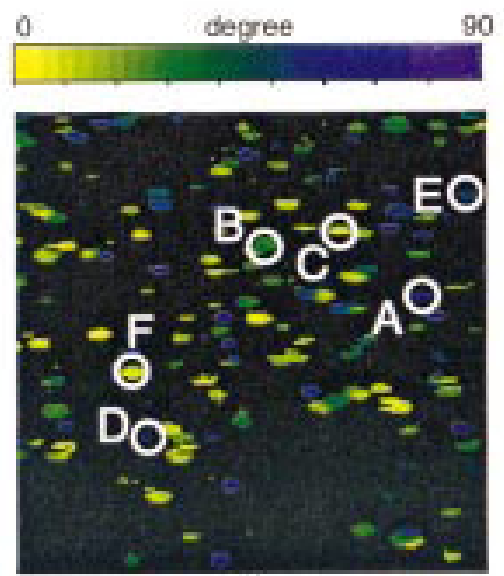

c)
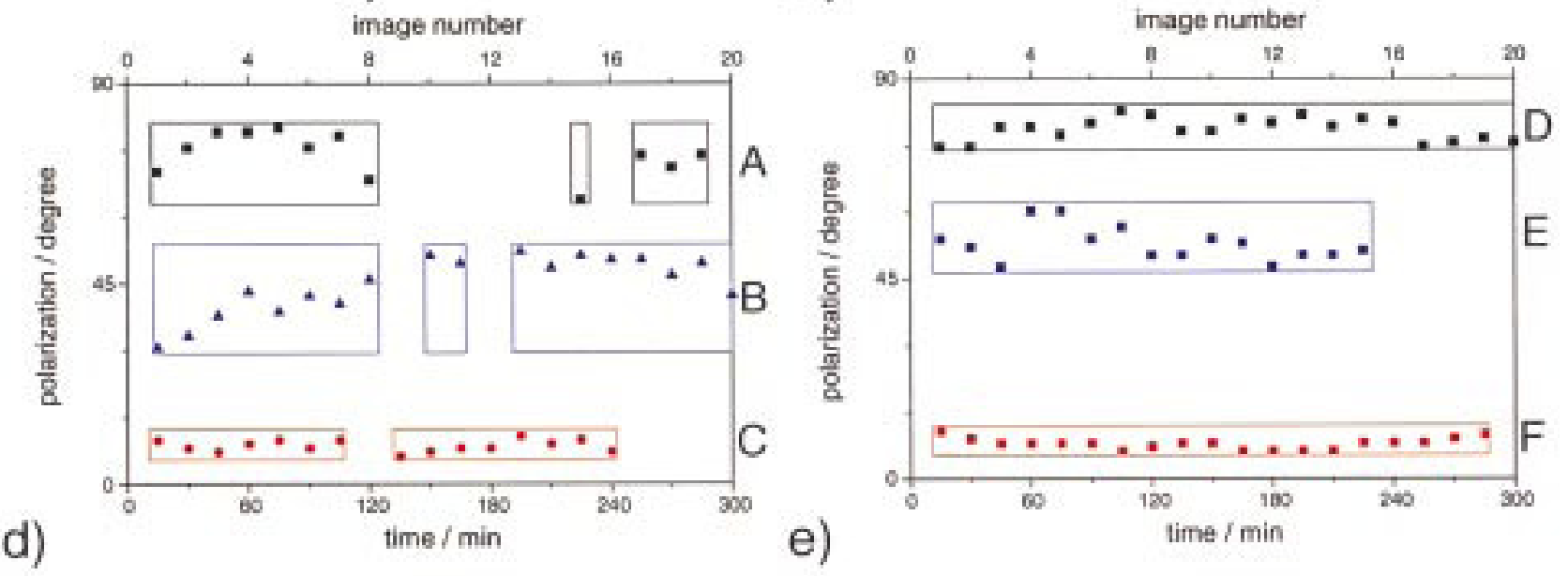

Figure 6. (a, b) Linear polarization components of fluorescence light in single-molecule images with circular polarized excitation. Both images were taken simultaneously with two independent photon counters. ${ }^{5}$ On-off transitions occurring during the scan (arrows) appear simultaneously in (a) and (b). (c) Polarization distribution calculated from (a) and (b), with the low-intensity regions of the image blanked out to better visualize the positions of the molecules. (d, e) Temporal dependence of the fluorescence polarization for single molecules [circles A-F in (c)], as deduced from a series of 20 images: (d) blinking, and (e) nonblinking molecules. For all of the molecules, the polarization does not change by $>15-20^{\circ}$ during the $5 \mathrm{~h}$ of observation, which excludes a molecular rotation as the reason for the on-off-on transitions.

histogram. For some molecules, $>300000$ counts were registered; taking the detection efficiency of $1-2 \%$ into account, this corresponds to at least $1.5 \times 10^{7}$ fluorescence photons at a fluorescence rate as great as $6 \times 10^{6} \mathrm{~s}$ for an excitation intensity of $I=300 \mu \mathrm{W} / \mu \mathrm{m}^{2}$. Taking into account the scanning process, where only a fraction $\left[1 \mu \mathrm{m}^{2} /(40 \mu \mathrm{m} \times 40 \mu \mathrm{m})=1 / 1600\right]$ of the image area is illuminated at any given time, the average excitation intensity is $I=1 / 1600 \times 300 \mu \mathrm{W} / \mu \mathrm{m}^{2}=0.19 \mu \mathrm{W} /$ $\mu \mathrm{m}^{2}$. The lifetime of the ensemble, determined as the $1 / \mathrm{e}$ decay time in Figure 4b, is $60 \mathrm{~min}$. The lifetime for single molecules (during continuous illumination), determined by the photon number and the rate from above, is $2.5 \mathrm{~s}$. The product of lifetime and intensity should be constant. Indeed, for single molecules it is similar to that of the ensemble, since $2.5 \mathrm{~s} \times$ $300 \mu \mathrm{W} / \mu \mathrm{m}^{2} \approx 60 \mathrm{~min} \times 0.19 \mu \mathrm{W} / \mu \mathrm{m}^{2}$.

From the histogram can be deduced a broad distribution of this total fluorescence yield. For some molecules, which bleached during the experiment, $<1 / 8$ of the counts of the bright and stable molecules were detected. This may simply be an effect of fundamental statistics: If the ensemble shows an exponential decay, then even if the molecules and their surroundings are identical (as in a homogeneous ensemble), most of the molecules must be less stable and bleach at the beginning of the experiment, while only few survive to the end. However, strong inhomogeneities between molecules or in their local environments can also be responsible for these widely differing total photon yields. Another reason for the different number of counts before bleaching is the dependence of the detection efficiency on the orientation of the molecules, which was not taken into account in the calculation of the minimum number of emitted photons above.

Blinking and Bleaching in Timeline Scans. To get a more quantitative insight into this effect, we repeatedly scanned intensity profiles $\mathrm{C}(x, t)$ above one line on the sample (Figure 5a). This view allowed us to follow the fluorescence intensity of several single molecules over a long period quasi-simultaneously. The scan profiles were $40 \mu \mathrm{m}$ long. The acquisition time for each line was $2.6 \mathrm{~s}$. In total, 768 lines were collected in $2000 \mathrm{~s}$. Molecules that are fluorescing constantly appear as a continuous line (E), whereas other molecules switch "off" and "on," on a time scale of seconds (D) to many minutes (A). Figure $5 \mathrm{~b}$ shows intensity vs time curves $\mathrm{C}\left(x_{i}, t\right)$ extracted from Figure 5a by summing the intensity over a range of $\Delta x=8$ pixels at different positions $\mathrm{A}-\mathrm{F}$, again showing the "inhomogeneity" in the behavior of the molecules. Jump heights of different intensities can be explained by different molecular orientations with respect to the optical axis. For some time, traces A and D show a second or third intensity level, perhaps 
from two or three molecules contributing to the fluorescence. Summing the intensities in Figure 5a over the scan lines yields Figure 5c. Although only a few molecules contribute to the fluorescence, the intensity decay curve compares well with the exponential decay curve (smooth curve) that would be roughly expected for a molecule ensemble.

Fluorescence Polarization. In another experiment we studied the polarization of the fluorescence light from single molecules, when they were excited by circular polarized light. The emission polarization for each spot directly corresponded to the molecular emission dipole component parallel to the object plane. Two images of both linear polarization components in the microscope were simultaneously acquired, as reported elsewhere. ${ }^{5}$ By calculating the inverse tangents from the relation of the intensities in the two detection channels for each pixel, we deduced an image of the polarization distribution. Figure 6 shows an image of the $x$ - and $y$-polarized light and the corresponding polarization image, where in the polarization images the low intensities of the fluorescence are blanked out. By comparing Figures $6 \mathrm{a}$ and $6 \mathrm{~b}$, we can see that molecules simultaneously blink and bleach in both polarization channels: Spots disappear and reappear at the same scan position (arrows). Figures $6 \mathrm{~d}$ and $6 \mathrm{e}$ show the time-dependence of the orientation of single molecules, which is extracted from a series of 20 polarization images. For all molecules, whether blinking (Figure 6d) or stably emitting (Figure 6e), the change of polarization over the $5 \mathrm{~h}$ is $<20^{\circ}$; for most molecules, it is $<15^{\circ}$. This very slow rotation of some molecules can be responsible for small and slow changes in intensity. However, since obviously the dye molecules make no strong rotation in the polymer film, then for our preparation, a rotation of the molecules as the origin for the blinking can be excluded on a time scale of hours. Consequently, another mechanism must be responsible for the long-time blinking.

\section{Discussion}

The observation of fluorescence intensity and polarization of single dye molecules over a period of seconds to several hours uncovers short- and long-time blinking, which cannot be detected in ensemble measurements. Our polarization measurements exclude fast rotation of the complete molecule as the reason for instantaneous intensity changes. However, very slow intensity changes, which are observed for very few molecules, can be explained by a slow molecular rotation, probably together with the sourrounding polymer strands. The question remains, as to the origin of the instantaneous blinking behavior. Several models have been discussed in the literature..$^{3,12,21}$ Meixner et al., ${ }^{3}$ investigating with a near-field microscope, attributed small translational shifts of rhodamine $6 \mathrm{G}$ in PVB to a molecular rotation. Xie and Dunn ${ }^{4}$ measured the molecular rotation of sulforhodamine 101 (dispersed on glass) on the time scale of a minute but found no rotation. Ruiter et al.,5 studying DiI (dioctadecyltetramethylindocarbocyanine) molecules in PMMA (poly(methyl methacrylate)), found a very slow molecular rotation on the time scale of $1 \mathrm{~h}$; they attributed blinking to temporary transitions to dark states or spectral jumps. In this study, a sudden rotation was found to be unlikely. In singlemolecule emission spectra, strong spectral shifts in the range of several nanometers were observed., ${ }^{4,22}$ Shifts in the excitation spectrum may explain one-step changes in the fluorescence intensity as well as slow changes. These spectral changes could be due to conformational changes of the molecules or of their surroundings. For the TDI molecules, spectral changes are expected for rotation of the substituents on the nitrogen atoms
(Figure 2). For free molecules, a specific orientation of the substituents in relation to the main molecular frame to form the $\pi$-system, which is responsible for the optical transition, is energetically favorable. ${ }^{23}$ In the PVB film, we must assume that the ability of the endgroups to rotate is strongly influenced by the individual local surroundings formed by the polymer strands. Some substituents might never have the chance to be in a favorable orientation with respect to the $\pi$-system because of limited space and thus would prevent an excitation by the laser. This could help account for the discrepancy between the number of fluorescing molecules observed and the number of molecules expected to be on the surface. Apart from spectral jumps, another possible explanation of the temporary "off" states could be temporary reactions with surrounding molecules. For example, the temporary presence of ${ }^{3} \mathrm{O}_{2}$ in the vicinity of the dye molecule could, by influencing the symmetry of the quantum mechanical levels of the molecule, reduce the excitation transition probability and consequently decrease the fluorescence intensity to a very small amount. After some time, which would vary stochastically between individual molecules because of fundamental statistics or different local surroundings, the oxygen could be released from the molecule and the fluorescence would reappear instantaneously. An experimental test of this model would be to study the dependence of the blinking behavior on the oxygen concentration. Recent results of such experiments show that the blinking is indeed strongly influenced by the surrounding oxygen concentration. ${ }^{24}$

\section{Conclusions}

TDI molecules, diluted in a PVB polymer film, were investigated over a span of $5 \mathrm{~h}$. Characteristic discrete longtime blinking and bleaching in the fluorescence demonstrates that the distribution of single TDI molecules can be imaged with our modular confocal microscope with high quality over a long time. No diffusion out of a range of $200 \mathrm{~nm}$ was observed during $5 \mathrm{~h}$. Some TDI molecules delivered $>1.5 \times$ $10^{7}$ fluorescence photons without bleaching. The fluorescence, summed for several molecules either in a series of 20 successively scanned images or in time-line scans, decayed in a nearly exponential way, as would be expected for a molecule ensemble. Polarization measurements of the fluorescence light with two parallel counters allowed us to follow molecular rotations by using circular polarized excitation light. For TDI in PVB, the polarization direction did not change by $>20^{\circ}$ over the of $5 \mathrm{~h}$, which excludes molecular rotation as the origin of the instantanous blinking. The influence of oxygen on the blinking and bleaching will be topic of a forthcoming paper.

Acknowledgment. A.H. and K.M. thank the German Fund of the Chemical Industry. Part of the image analysis was performed with a program written by J. Ferber. This project is supported by the Deutsche Forschungsgemeinschaft.

\section{References and Notes}

(1) Betzig, E.; Chichester, R. J. Science 1993, 262, 1422.

(2) Ambrose, W. P.; Goodwin, P. M.; Martin, J. C.; Keller, R. A. Phys. Rev. Lett. 1994, 72, 160.

(3) Meixner, A. J.; Zeisel, D.; Bopp, M. A.; Tarrach, G. Rev. Sci. Instrum. 1995, 66, 3569-3575.

(4) Xie, X. S.; Dunn, R. C. Science 1994, 265, 361.

(5) Ruiter, A. G. T.; Veerman, J. A.; Garcia-Parajo, M. F.; van Hulst, N. F. J. Phys. Chem. 1997, 101, 7318.

(6) Nie, S.; Chiu, D. T.; Zare, R. N. Science 1994, 266, 1018.

(7) Schmidt, Th.; Schütz, G. J.; Baumgartner, W.; Gruber, H. J.; Schindler, H. J. Phys. Chem. 1995, 99, 17662.

(8) Eigen, M.; Rigler, R. Proc. Natl. Acad. Sci. USA 1994, 91, 5740. 
(9) Shera, E. B.; Seitzinger, N. K.; Davis, L. M.; Keller, R. A.; Soper, S. A. Chem. Phys. Lett. 1990, 174, 553.

(10) Basché, Th.; Moerner, W. E.; Orrit, M.; Wild, U. P., Eds.; Single Molecule Optical Detection, Imaging and Spectroscopy; VCH: Weinheim, 1997.

(11) Jasny, J.; Sepiol, J.; Th. Irngartinger, Traber, M.; Renn, A.; Wild, U. P.; Rev. Sci. Instrum. 1997, 67, 1425.

(12) Lu, H. P.; Xie, X. S. Nature 1997, 385, 143.

(13) Trautman, J. K.; Macklin, J. J. Chem. Phys. 1997, 205, 221.

(14) Basché, Th.; Ambrose, W. P.; Moerner, W. E. J. Opt. Soc. Am. B $1992,9,829$.

(15) Funatsu, T.; Harada, Y.; Tokunaga, M.; Saito, K.; Yanagida, T. Nature 1995, 374, 555-559.

(16) Ha, T.; Enderle, Th.; Ogletree, D. F.; Chemla, D. S.; Selvin, P. R.; Weiss, S. Proc. Natl. Acad. Sci. USA 1996, 93, 6264-6268.
(17) Göhde, W.; Tittel, J.; Basché, Th.; Bräuchle, C.; Fischer, U. C.; Fuchs, H. Rev. Sci. Instrum. 1997, 68, 2466-2474.

(18) Holtrup, F. O.; Müller, G. R. J.; Quante, H.; De Feyter, S.; De Schryver, F. C.; Müllen, K. Chem. Eur. Lett. 1997, 3, 219-225.

(19) Mais, S.; Tittel, J.; Basché, Th.; Bräuchle, C.; Göhde, W.; Fuchs, H.; Müller, G.; Müllen, K. J. Phys. Chem. A 1997, 101, 8435-8440. (20) Plakhotnik, T.; Moerner, W. E.; Palm, V.; Wild, U. P. Opt. Commun. 1995, 114, 83

(21) Moerner, W. E. Science 1997, 277, 1059-1060

(22) Seisenberger, G.; Göhde, W.; Fischer, U. C.; Fuchs, H.; Bräuchle, Ch. manuscript in preparation.

(23) Calculations from R. Ellerbrake, Westfälische Wilhelms-Universität Münster.

(24) Panzer, O.; Göhde, W.; Fischer, U. C.; Fuchs, H.; Tittel, J.; Basché, Th.; Herrmann, A.; Müllen, K. manuscript in preparation. 